\section{Associação entre indicadores antropométricos de obesidade e risco coronariano em adultos na cidade de Salvador, Bahia, Brasil*}

\section{Resumo}

Objetivo: O estudo teve como objetivo determinar a associação entre os diversos indicadores de obesidade e risco coronariano elevado (RCE) em adultos na cidade de Salvador-BA. Métodos: O desenho foi de corte transversal, com amostra composta por 968 adultos de 30-74 anos de idade, sendo 391 (40.4\%) homens. A análise constou da regressão logística, sendo calculadas as Odds Ratio (OR) entre o índice de conicidade (índice $\mathrm{C}$ ), índice de massa corporal (IMC), razão circunferência cinturaquadril (RCCQ), circunferência de cintura (CC) e RCE. Utilizou-se intervalo de confiança a 95\%. Resultados: Após ajustamento por idade, as OR encontradas para homens foram: a) RCCQ: 5.81 (3.00-11.23), b) índice C: 5.52 (2.94-10.36), c) CC: 4.37 (2.318.26), d) IMC: 3.04 (1.62-5.73). Para mulheres dos 30 aos 49 anos e 50 aos 74 anos as OR encontradas foram, respectivamente: a) RCCQ: 7.85 (2.15-28.69) e 1.81 (0.98-3.36); b) IMC: 7.28 (1.61-32.97) e 1.09 (0.61-1.96); c) índice C: 6.88 (1.89-25.11) e 2.89 (1.585.27); d) CC: 6.41 (2.09-19.65) e 1.38 (0.772.50). Conclusões: Os resultados demonstram que, entre homens e mulheres de 3049 anos, todos os indicadores de obesidade apresentam forte associação com RCE, destacando-se entre os homens os indicadores de obesidade central, RCCQ e IC, enquanto que para as mulheres entre $50 \mathrm{e}$ 74 anos o índice $\mathrm{C}$ é o melhor indicador.

Palavras-chaves: Indicadores antropométricos de obesidade. Risco coronariano. Obesidade abdominal.

\section{Francisco José Gondim Pitanga' \\ Ines Lessa ${ }^{2}$ \\ ' Departamento de Educação Física, Faculdade de Educação, Universidade Federal da Bahia \\ ${ }^{2}$ Insituto de Saúde Coletiva, Universidade Federal da Bahia}

\footnotetext{
* Trabalho financiado pela Secretaria de Vigilância em Saúde, Ministério da Saúde do Brasil e Banco Mundial.

Correspondência: Francisco José Gondim Pitanga. Av. Luiz Tarquínio Pontes, 600 - Centro - Lauro de Freitas, BA CEP: 42700-000. E-mail: pitanga@lognet.com.br
} 
Abstract

Objective: To determine the association between the many anthropometric indicators of obesity and high coronary risk (HCR). Methods: This is a cross-sectional study. Its population comprised 968 adults, 30-74 years of age, of which 391 (40.4\%) were men. Logistic regression analysis was used and the Odds Ratio (OR) was calculated between the conicity index (C index), body mass index (BMI), waist-to-hip ratio (WHR), waist circumference (WC), and HCR. A 95\% confidence interval was utilized. Results: The OR between obesity indicators and HCR, adjusted by age, in men were: a) WHR - 5.81 (3.00-11.23), b) C index - 5.52 (2.94-10.36), c) WC - 4.37 (2.318.26), d) BMI - 3.04 (1.62-5.73). In women 30-49 years old and 50-74 years old the OR adjusted by age, respectively, were: a) WHR - 7.85 (2.15-28.69) and 1.81 (0.98-3.36), b) BMI - 7.28 (1.61-32.97) and 1.09 (0.61-1.96), c) Cindex - 6.88 (1.89-25.11) and 2.89 (1.585.27), d) WC - 6.41 (2.09-19.65) and 1.38 (0.77-2.50). Conclusion: Both in men and women, 30-49 years old, all obesity indicators are strongly associated with HCR. However, WHR and C index were found to be the most effective in male individuals, while $C$ index was the best indicator in women from 50 to 74 years of age.

Keywords: Anthropometric Indices Of Obesity. Coronary Risk. Abdominal Obesity.

\section{Introdução}

A obesidade considerada na atualidade uma epidemia em vários países industrializados é um dos maiores problemas de saúde das sociedades contemporâneas, estando relacionada a elevadas prevalências e incidências de doenças cardiovasculares (hipertensão, doença arterial coronariana e doenças cerebrovasculares), diabetes e cálculos biliares, entre outras ${ }^{1,2}$.

A doença arterial coronariana persiste como a mais importante causa de mortes entre adultos no mundo. Fatores de risco incluem pressão arterial, tabagismo, colesterol total, LDL-C, HDL-C e diabetes. Fatores como obesidade, sedentarismo, hipertrofia do ventrículo esquerdo e história familiar prematura de eventos coronarianos também têm sido considerados na definição do risco de doença arterial coronariana (DAC) ${ }^{3}$.

O papel da obesidade generalizada como fator de risco cardiovascular é controverso; no entanto, a melhor explicação para a associação entre obesidade e doença cardíaca isquêmica é de que esta ocorreria em um subgrupo de obesos, ou seja, nas pessoas que apresentassem adiposidade localizada na região abdominal ou central, mesmo na ausência da obesidade generalizada ${ }^{4}$.

Diversos estudos têm sido realizados para verificar a associação entre indicadores antropométricos de obesidade e risco coronariano elevado (RCE). A maioria deles demonstra que os indicadores de obesidade central são mais associados ao RCE do que os indicadores de obesidade total ${ }^{5-8}$.

Existem diversos indicadores de obesidade total e central: a obesidade central pode ser medida pela espessura de dobras cutâneas no tronco e nas extremidades; por medidas de circunferências de cintura e quadril; por tomografia computadorizada, ultrassonografia abdominal e ressonância magnética nuclear'. Mais recentemente, no início da década de $90^{10}$ foi proposto o índice de conicidade (índice $\mathrm{C}$ ) como indicador para avaliação da obesi- 
dade e distribuição da gordura corporal. Este índice utiliza como variáveis o peso, a estatura e a circunferência da cintura.

A razão circunferência cintura-quadril (RCCQ), apesar de ser sensível para a distribuição da gordura corporal, poderá apresentar problemas em virtude da provável variação conjunta nas circunferências de quadril e cintura, durante o processo de redução do peso corporal ${ }^{10}$. Por outro lado, sugere-se que a medida da circunferência da cintura (CC) está mais fortemente associada ao nível de tecido adiposo abdominal visceral do que à RCCQ, utilizando-se a tomografia computadorizada como método de referência ${ }^{11}$. Além disto, pesquisas demonstram que a circunferência da cintura pode ter vantagens sobre outras medidas de obesidade na predição do risco cardiovascular ${ }^{12}$, porém novos estudos são necessários para confirmar esta hipótese.

Para avaliação da obesidade generalizada, o indicador mais utilizado é o índice de massa corporal (IMC) que não reúne condições para avaliação da distribuição da gordura corporal, além de nem sempre estimar de forma correta padrões de obesidade em alguns subgrupos populacionais, como, por exemplo, atletas.

O principal objetivo deste estudo foi determinar e comparar a magnitude da associação entre o índice C, RCCQ, CC e IMC com RCE em adultos de ambos os sexos na cidade de Salvador-BA.

\section{Métodos}

Estudo de corte transversal realizado em Salvador, Brasil, em 2000, em subgrupo de participantes do projeto "Monitoramento das Doenças Cardiovasculares e do Diabetes no Brasil" (MONIT), desenvolvido pela equipe de doenças crônicas não transmissíveis do Instituto de Saúde Coletiva (ISC) da Universidade Federal da Bahia (UFBA) e financiado pelo Ministério da Saúde do Brasil. Para a coleta dos dados foi utilizado o censo domiciliar realizado por investigadores do projeto "Bahia Azul”, em desenvolvimento por pesquisadores do ISC-UFBA e direcionado a outros objeti$\operatorname{vos}^{13,14}$.

\section{Amostra}

A amostra original foi constituída por 2. 297 adultos com idade entre 20 a 74 anos de idade, sendo 1.025 do sexo masculino e 1.272 do sexo feminino. Como o indicador de risco coronariano utilizado no presente estudo foi construído com base em população de 30 a 74 anos, a amostra foi reduzida para 1.654 adultos, sendo 711 homens e 943 mulheres. Para o presente estudo foi utilizado subgrupo deste grupo etário constituído por pessoas que completaram todo o protocolo proposto, totalizando 391 homens e 577 mulheres, com idade entre 30 e 74 anos, o que equivale a $55 \%$ de participantes do sexo masculino e $61 \%$ do sexo feminino em relação ao banco de dados original das faixas etárias em contexto. Os procedimentos para o delineamento amostral, além do estudo para identificar que as perdas não influenciaram nos resultados do trabalho foram previamente descritos em detalhes ${ }^{15}$.

\section{Variáveis de estudo}

Foram utilizadas as seguintes variáveis: idade; sexo; estatura; peso; circunferência da cintura; circunferência do quadril; tabagismo; glicemia; colesterol total; HDLC; pressão arterial sistólica (PAS); pressão arterial diastólica (PAD); escolaridade; raça.

Utilizando algumas destas variáveis, foi construído um indicador de risco coronariano para representar, em conjunto, os fatores de risco cardiovascular analisados simultaneamente.

\section{Construção do indicador de risco coronariano}

Com base no acompanhamento por 12 anos de 2.489 homens e 2.856 mulheres de 30 a 74 anos, da coorte de Framingham, 
Wilson et al. ${ }^{3}$ construíram o algorítimo para medida do risco coronariano que serviu de modelo para o estudo de Salvador, Brasil. Durante o acompanhamento da coorte, 383 homens e 227 mulheres desenvolveram DAC. Através da utilização do modelo de regressão de Cox, os autores elaboraram uma tabela de pontuação (algoritmo) com a inclusão das variáveis por eles selecionadas (idade, PAS, PAD, colesterol total, HDL-C, tabagismo e diabetes). Para cada variável a pontuação poderia ser positiva, quando considerada fator de risco, ou negativa, quando considerada fator de proteção. Utilizaram as seguintes pontuações:

- para idade : < 35 anos (-1 para homens e -9 para mulheres); 35 a 39 anos (0 para homens e -9 para mulheres); 40 a 44 anos ( 1 para homens e 0 para mulheres); 45 a 49 anos (2 para homens e 3 para mulheres); 50 a 54 anos (3 para homens e 6 para mulheres); 55 a 59 anos (4 para homens e 7 para mulheres); 60 a 64 anos (5 para homens e 8 para mulheres); 65 a 69 anos (6 para homens e 8 para mulheres) e 70 a 74 anos ( 7 para homens e 8 para mulheres);

- para o colesterol total: < $160 \mathrm{mg} / \mathrm{dl}$ (-3 pontos para homens e -2 pontos para mulheres); 160 a $199 \mathrm{mg} / \mathrm{dl}$ (0 ponto para homem e 0 ponto para mulheres); 200 a $239 \mathrm{mg} / \mathrm{dl}$ (1 ponto para homem e 1 ponto para mulheres); 240 a $279 \mathrm{mg} /$ dl ( 2 pontos para homens e 2 pontos para mulheres); > $280 \mathrm{mg} / \mathrm{dl}$ (3 pontos para homens e 3 pontos para mulheres).

- para o $H D L-C$-: $<35 \mathrm{mg} / \mathrm{dl}$ (2 pontos para homens e 5 pontos para mulheres); 35 a $44 \mathrm{mg} / \mathrm{dl}$ (1 ponto para homens e 2 pontos para mulheres); 45 a $49 \mathrm{mg} / \mathrm{dl}$ (0 ponto para homens e 1 ponto para mulheres); 50 a $59 \mathrm{mg} / \mathrm{dl}(0$ ponto para homens e 0 ponto para mulheres); > $60 \mathrm{mg} / \mathrm{dl}$ (-2 pontos para homens e -3 pontos para mulheres).

- para $P A S:<120 \mathrm{mmHg}$ (0 ponto para homens e -3 pontos para mulheres);
120 a $129 \mathrm{mmHg}$ (0 ponto para homens e mulheres); 130 a $139 \mathrm{mmHg}$ (1 ponto para homens e mulheres); 140 a $159 \mathrm{mmHg}$ (2 pontos para homens e mulheres); > $160 \mathrm{mmHg}$ (3 pontos para homens e mulheres).

- para $P A D:<80 \mathrm{mmHg}$ (0 ponto para homens e -3 pontos para mulheres); 80 a $84 \mathrm{mmHg}$ (0 ponto para homens e mulheres); 85 a $89 \mathrm{mmHg}$ (1 ponto para homens e 0 ponto para mulheres); 90 a $99 \mathrm{mmHg}$ ( 2 pontos para homens e mulheres); > $100 \mathrm{mmHg}$ (3 pontos para homens e mulheres).

- para diabetes: glicemia plasmática após 12 horas de jejum $\geq 126 \mathrm{mg} / \mathrm{dl}$ (2 pontos para homens e 4 quatro pontos para mulheres); glicemia plasmática após 12 horas de jejum < $126 \mathrm{mg} / \mathrm{dl}$ ( 0 ponto para homens e mulheres).

- para tabagismo: qualquer cigarro fumado no último ano (2 pontos para homens e mulheres); nenhum cigarro fumado no último ano (0 ponto para homens e mulheres).

Para determinação dos pontos, utilizaram o coeficiente beta dos modelos de análises de regressão de $\mathrm{Cox}^{3}$.

Cada participante da amostra do presente estudo teve sua pontuação calculada com base no algoritmo supracitado. Para identificação do RCE, as somas das pontuações foram colocadas em ordem crescente e determinados os percentis, selecionando-se a soma da pontuação referente ao percentil 80 como de risco coronariano elevado, que correspondeu a 8 pontos para o sexo masculino e 10 pontos para o sexo feminino.

\section{Coleta dos dados}

Todos os participantes da pesquisa foram entrevistados em domicílio para coleta dos dados demográficos, e tiveram a PAS e PAD medidas por 6 (seis) vezes, as primeiras 3 consecutivamente, 30' após início da entrevista, e as 3 seguintes após intervalo de 20', braço esquerdo, sentado, 
bexiga vazia, sem ter fumado, tomado café ou álcool nos 30' precedentes às tomadas. A cintura foi medida estando o sujeito com o mínimo de roupa possível, na distância média entre a última costela flutuante e a crista ilíaca, enquanto o quadril foi medido com a fita métrica passando pelos trocânteres femurais (duas medidas) de cada circunferência. Peso e estatura foram medidos no Centro de Saúde de cada bairro pela própria equipe do estudo (duas medidas). $\mathrm{O}$ índice $\mathrm{C}$ foi determinado através das medidas de peso, estatura e circunferência da cintura utilizando-se a equação matemática ${ }^{10}$ :

Índice $C=\frac{\text { Circunferência Cintura }(\mathrm{m})}{0,109 \sqrt{\frac{\text { Peso Corporal }(\mathrm{kg})}{\text { Estatura }(\mathrm{m})}}}$

O IMC foi determinado pela divisão do peso/estatura ${ }^{2}$. A RCCQ foi determinada pela divisão da circunferência da cintura pela circunferência do quadril. A coleta do sangue para os exames bioquímicos, após 12 horas de jejum, foi realizada no Centro de Saúde de suporte ao projeto. Foram realizadas dosagens de colesterol total (método Trinder enzimático), HDL-C (método Labtest) e glicemia (método Trinder enzimático). As técnicas e métodos para os exames bioquímicos foram padronizados pela Sociedade Brasileira de Patologia Clínica. Todos os participantes do estudo ou seus responsáveis assinaram termo de consentimento concordando em participar da pesquisa. O projeto foi apresentado ao Comitê de Ética do Conselho Regional de Medicina do Estado da Bahia, sendo aprovado na íntegra.

\section{Instrumentos Utilizados}

Para medida da pressão arterial, foi utilizado tensiômetro eletrônico (OMRON, modelo HEM-705CP). A estatura foi medida com estadiômetro inglês, tipo "Leicesters", acoplado a uma base para medida de estatura em campo, fabricado pela Child Growth Foundation. O peso corporal foi medido em balanças padrão, tipo "banheiro", novas e aferidas no início e periodicamente pelo INMETRO, com certificado próprio referindo margem de erro de \pm 100 gr. Para medida de circunferência de cintura e quadris, foi utilizada fita métrica metálica e flexível, marca Starrett, com definição de medida de $0,1 \mathrm{~cm}$.

\section{Procedimentos de Análise}

Foram propostos quatro modelos de regressão logística, tendo o RCE como variável dependente em todos eles. O RCE foi introduzido em cada modelo de forma dicotômica tendo como ponto de corte os valores de 8 e 10 para homens e mulheres, respectivamente. As variáveis independentes principais foram introduzidas em cada modelo de regressão logística, sendo utilizados o índice C, RCCQ, IMC e CC. Todas as variáveis independentes principais foram introduzidas nos modelos também de forma dicotômica, tendo como pontos de corte os seguintes valores para homens e mulheres, respectivamente: índice $\mathrm{C}, 1,25$ e 1,18; RCCQ, 0,92 e 0,83; CC, $88 \mathrm{~cm}$ e $83 \mathrm{~cm}$ e IMC, $24 \mathrm{~kg} / \mathrm{m}^{2}$ e $26 \mathrm{~kg} / \mathrm{m}^{28}$. Em todos os modelos foram introduzidas como covariáveis a idade, a escolaridade e a raça.

$\mathrm{Na}$ seqüência, foi realizada estratificação para análise de modificação de efeito e confundimento para cada um dos modelos propostos. A análise para modificação de efeito foi realizada através da observação das medidas pontuais estratoespecíficas e de seus intervalos de confiança. Quando a medida pontual de um fator, em determinado estrato específico, não estava contida no intervalo de confiança do outro fator no mesmo estrato, isto indicava modificação de efeito. Foi utilizado intervalo de confiança de $95 \%$ pelo método de Mantel-Haenzel. A análise para confundimento foi feita comparando-se a OR entre a associação bruta e ajustada pelos possíveis confundidores. Utilizou-se como parâmetro para identificar a diferença entre as associações o valor de $20 \%$. 
Foram considerados como potenciais modificadores de efeito e confundimento da associação entre indicadores antropométricos de obesidade e RCE as variáveis idade, escolaridade e raça, todas introduzidas na modelagem de forma dicotômica: idade $=0$ se $<50$ anos e idade $=1$ se $\geq 50$ anos; escolaridade $=0$ se universitário, $2^{\circ}$ ou $1^{\circ}$ grau completo e escolarida$\mathrm{de}=1$ se analfabeto ou nunca foi à escola, porém lê e escreve; raça $=0$ se branco $\mathrm{e}$ raça $=1$ se negro, mulato, moreno e outros.

Logo após foi realizada a análise de regressão logística, partindo-se do modelo completo com termos-produto. A modelagem foi realizada com procedimento backward. Foram-se retirando os termosproduto para análise de modificação de efeito através do teste da razão de máxima verossimilhança. Em seguida, a análise de regressão logística foi aplicada sem os termos-produto, partindo-se do modelo completo e retirando-se uma a uma as possíveis variáveis de confundimento. Variáveis modificadoras de efeito foram as que apresentaram resultados estatisticamente significantes baseando-se no teste da razão de máxima verossimilhança, correspondendo a comparações entre os modelos saturados e reduzidos, nos quais termos-produtos relativos a cada covariável haviam sido retirados. Variáveis de confundimento foram aquelas que, quando retiradas do modelo, causaram alteração igual ou superior a $20 \%$ na medida pontual de associação entre a variável RCE e os indicadores antropométricos de obesidade estudadas ${ }^{16}$. Finalmente, estimouse a OR entre os indicadores antropométricos de obesidade e RCE através do modelo que melhor explicou esta associação. Não foi evidenciada no processo de modelagem a presença de modificação de efeito ou confundimento por raça ou escolaridade. Desta forma, entre os homens, o melhor modelo foi o ajustado por idade, considerando-se que esta variável foi forte confundidora em todas as análises realizadas. Entre as mulheres, a idade foi con- siderada tanto confundidora quanto modificadora de efeito. Assim, optou-se pela estratificação, com a análise sendo feita em dois grupos separadamente: 30-49 anos e 50-74 anos. Empregou-se o programa estatístico “STATA, versão 7.0”.

\section{Resultados}

As características da amostra estão demonstradas na Tabela 1. Observa-se que, apesar de não existirem diferenças estatisticamente significativas entre homens e mulheres nas variáveis escolaridade, raça e risco coronariano, a maioria dos participantes da amostra apresenta baixo risco coronariano e não são brancos. Observamse diferenças estatisticamente significativas entre homens e mulheres nas variáveis: peso corporal, estatura, circunferência da cintura e do quadril, TAS, TAD, colesterol total, HDL-C, índice C, IMC, RCCQ e CC.

Na Tabela 2, encontram-se as OR entre os indicadores antropométricos de obesidade e risco coronariano em indivíduos do sexo masculino, notando-se que a RCCQ e índice C são os indicadores antropométricos de obesidade que apresentam maior associação com risco coronariano em pessoas do sexo masculino.

Na Tabela 3, aparecem as OR entre os indicadores antropométricos de obesidade e risco coronariano em indivíduos do sexo feminino. No grupo etário das mulheres jovens, todos os indicadores de obesidade analisados apresentam associação com risco coronariano. Importante ressaltar que em mulheres a partir de 50 anos de idade as associações são mais fracas e apenas o índice $C$ apresenta associação estatisticamente significativa com risco coronariano.

\section{Discussão}

Diversos estudos têm demonstrado associação entre indicadores antropométricos de obesidade e risco cardiovascular $^{5,7,17-20}$, porém existem controvérsias sobre qual destes indicadores apresenta 
Tabela 1 - Média, desvio padrão, valores mínimos, máximos e percentuais das variáveis analisadas no estudo. Salvador-Brasil, 2006.

Table 1 - Average, standard deviation, minimal and maximum values and variable percentages analyzed in the study. Salvador-Brazil, 2006.

\begin{tabular}{|c|c|c|c|}
\hline & $\begin{array}{l}\text { Homens } \\
(n=391)\end{array}$ & $\begin{array}{l}\text { Mulheres } \\
(n=577)\end{array}$ & p ou $\chi^{2}$ \\
\hline IDADE (anos) & $\begin{array}{c}45,34 \pm 10,44 \\
(30,0-73,0)\end{array}$ & $\begin{array}{c}45,73 \pm 11,64 \\
(30,0-74,0)\end{array}$ & 0,59 \\
\hline PESO (Kg) & $\begin{array}{c}68,91 \pm 12,30 \\
(39,10-124,50)\end{array}$ & $\begin{array}{c}64,20 \pm 13,84 \\
(37,00-143,60)\end{array}$ & 0,00 \\
\hline ESTATURA (m) & $\begin{array}{c}1,68 \pm 0,07 \\
(144,0-191,0)\end{array}$ & $\begin{array}{c}1,55 \pm 0,07 \\
(100,0-181,0)\end{array}$ & 0,00 \\
\hline CINTURA $(\mathrm{cm})$ & $\begin{array}{l}85,64 \pm 10,11 \\
(62,0-122,80)\end{array}$ & $\begin{array}{c}82,68 \pm 12,19 \\
(43,80-129,30)\end{array}$ & 0,00 \\
\hline QUADRIL (cm) & $\begin{array}{l}93,73 \pm 7,46 \\
71,9-119,0\end{array}$ & $\begin{array}{c}99,72 \pm 10,00 \\
77,5-144,0\end{array}$ & 0,00 \\
\hline PAS (mmHg) & $\begin{array}{c}130,6 \pm 22,8 \\
(81,5-238,2)\end{array}$ & $\begin{array}{r}124,8 \pm 24,6 \\
(82,2-229,6)\end{array}$ & 0,00 \\
\hline PAD $(\mathrm{mmHg})$ & $\begin{array}{l}81,1 \pm 14,6 \\
(55-143,6)\end{array}$ & $\begin{array}{c}78,2 \pm 13,2 \\
(50-139)\end{array}$ & 0,00 \\
\hline COLESTEROL TOTAL (mg/dl) & $\begin{array}{c}220,6 \pm 52,0 \\
(102-522)\end{array}$ & $\begin{array}{c}232,3 \pm 52,5 \\
(123-468)\end{array}$ & 0,00 \\
\hline $\mathrm{HDL}-\mathrm{C}(\mathrm{mg} / \mathrm{dl})$ & $\begin{array}{c}48,5 \pm 13,1 \\
(24-114)\end{array}$ & $\begin{array}{c}51,5 \pm 13,5 \\
(26-98)\end{array}$ & 0,00 \\
\hline GLICEMIA (mg/dl) & $\begin{array}{c}90,5 \pm 29,2 \\
(61-308)\end{array}$ & $\begin{array}{c}92,0 \pm 34,4 \\
(59-318)\end{array}$ & 0,48 \\
\hline Índice C & $\begin{array}{c}1,23 \pm 0,07 \\
(1,01-1,45)\end{array}$ & $\begin{array}{c}1,18 \pm 0,09 \\
(0,59-1,63)\end{array}$ & 0,00 \\
\hline IMC (kg/m²) & $\begin{array}{c}24,4 \pm 3,85 \\
(15,94-36,29)\end{array}$ & $\begin{array}{c}26,54 \pm 5,39 \\
(15,57-52,74)\end{array}$ & 0,00 \\
\hline $\mathrm{RCCQ}$ & $\begin{array}{c}0,91 \pm 0,07 \\
(0,73-1,25)\end{array}$ & $\begin{array}{l}0,83 \pm 0,08 \\
(0,43-1,13)\end{array}$ & 0,00 \\
\hline $\mathrm{CC}(\mathrm{cm})$ & $\begin{array}{c}85,6 \pm 10,1 \\
(62,0-122,8)\end{array}$ & $\begin{array}{c}82,7 \pm 12,2 \\
(43,8-129,3)\end{array}$ & 0,00 \\
\hline RISCO CORONARIANO & & & \\
\hline Não elevado & $76 \%$ & $79 \%$ & \\
\hline Elevado & $24 \%$ & $21 \%$ & 0,61 \\
\hline ESCOLARIDADE & & & \\
\hline $\begin{array}{l}\text { Analfabeto/nunca foi à escola, } \\
\text { porém lê e escreve }\end{array}$ & $49 \%$ & $54 \%$ & \\
\hline $1^{\circ}$ grau & $46 \%$ & $43 \%$ & \\
\hline $2^{\circ}$ grau e universitário & $5 \%$ & $3 \%$ & 0,66 \\
\hline RAÇA & & & \\
\hline Brancos & $24 \%$ & $26 \%$ & \\
\hline Negros e outros & $76 \%$ & $74 \%$ & 0,74 \\
\hline
\end{tabular}

Valores contínuos foram comparados através do teste " $\mathrm{t}$ " de student para amostras independentes e valores percentuais através do teste qui-quadrado; PAS, pressão arterial sistólica; PAD, pressão arterial distólica; HDL-C, lipoproteína de alta densidade; índice C, índice de conicidade, IMC, índice de massa corporal; RCCQ, razão circunferência cintura-quadril; CC, circunferência da cintura; $\chi^{2}$, teste do qui-quadrado.

Continuing values were compared through student's $t$-test for independent samples and percent values through chi-square test; PAS $=$ systolic blood pressure; $P A D=$ diastolic arterial pressure; $H D L-C$ high-density lipoprotein; índice $C=$ conicity index; IMC = body mass index; $R C C Q=$ waist-to-hip ratio, $C C=$ waist circumference; $\chi^{2}$ chi-square test 
Tabela 2 - Associação entre indicadores antropométricos de obesidade e risco coronariano ajustados por idade. Salvador-Brasil, 2006.

Table 2 - Association between obesity anthropometric indicators and coronary risk adjusted by age. Salvador-Brazil, 2006.

\begin{tabular}{ccc}
\hline $\begin{array}{c}\text { INDICADORES ANTROPOMÉTRICOS } \\
\text { DE OBESIDADE }\end{array}$ & OR & IC (95\%) \\
\hline RCCQ & 5,81 & $3,00-11,23$ \\
Índice C & 5,52 & $2,94-10,36$ \\
CC & 4,37 & $2,31-8,26$ \\
IMC & 3,04 & $1,62-5,73$ \\
\hline
\end{tabular}

OR, Odds ratio; índice $C$, índice de conicidade; IMC, índice de massa corporal; $R C C Q$, razão circunferência cintura-quadril; $C C$ circunferência da cintura; IC, intervalo de confiança,

$O R=$ Odds ratio; Índice $C=$ conicity index; $I M C=$ body mass index; $R C C Q=$ waist-to-hip ratio, $C C$ = waist circumference; $I C$ confidence interval

Tabela 3 - Associação entre indicadores antropométricos de obesidade e risco coronariano controlados por idade. Salvador-Brasil, 2006.

Table 3 - Association between obesity anthropometric indicators and coronary risk controlled by age. Salvador-Brazil, 2006.

\begin{tabular}{ccccc}
\hline INDICADORES ANTROPOMÉTRICOS & \multicolumn{2}{c}{ MULHERES } & \multicolumn{2}{c}{ MULHERES } \\
DE OBESIDADE & \multicolumn{2}{c}{$30-49$ anos $(\mathrm{n}=388)$} & \multicolumn{2}{c}{$50-74$ anos ( $=189)$} \\
& OR & IC (95\%) & OR & IC (95\%) \\
\hline RCCQ & 7,85 & $2,15-28,69$ & 1,81 & $0,98-3,36$ \\
IMC & 7,28 & $1,49-32,97$ & 1,09 & $0,61-1,96$ \\
Índice C & 6,88 & $1,89-25,11$ & 1,91 & $1,04-3,53$ \\
CC & 6,41 & $2,09-19,65$ & 1,38 & $0,77-2,50$ \\
\hline
\end{tabular}

$\mathrm{OR}$, Odds ratio; índice C, índice de conicidade; IMC, índice de massa corporal; $\mathrm{RCCQ}$, razão circunferência cintura-quadril; $\mathrm{CC}$, circunferência da cintura; $\mathbf{I C}$, intervalo de confiança,

$O R=$ Odds ratio; Indice $C=$ conicity index; $I M C=$ body mass index; $R C C Q=$ waist-to-hip ratio, $C C=$ waist circumference; $I C$ confidence interval

maior associação com o risco coronariano.

No presente estudo, a RCCQ e o índice $\mathrm{C}$ foram os indicadores de obesidade que apresentaram maior associação com risco coronariano entre pessoas do sexo masculino.

Resultados contrários foram encontrados em homens indianos ${ }^{6}$, nos quais a CC e o IMC apresentaram maior associação com fatores de risco coronariano do que o índice $\mathrm{C}$.

Existem divergências entre resultados de estudos realizados na perspectiva de identificar a associação do índice $\mathrm{C}$ e outros indicadores antropométricos de obesidade com fatores de risco cardiovascular. Alguns autores ${ }^{5,21}$ observaram que o índice $\mathrm{C}$ é inferior aos outros índices de obesi- dade abdominal para ser utilizado como fator de risco cardiovascular e não apresenta vantagens sobre a RCCQ na avaliação da distribuição da gordura corporal. Outros ${ }^{17,20,22}$ afirmam que o índice $C$ é mais fortemente associado aos fatores de risco cardiovasculares do que diversos índices de obesidade, contribuindo para a prática clínica na detecção de risco para doenças coronarianas.

Em recente estudo ${ }^{7}$ utilizando como amostra 212 homens hindus demonstrou-se que o índice $C$ foi o indicador antropométrico de obesidade que melhor se associou com diversos fatores de risco cardiovascular, entre eles colesterol total, glicemia, triglicérides, HDL-C, LDL-C e VLDL-C.

Outros autores, ao estudarem a associ- 
ação entre CC e IMC com fatores de risco coronariano, demonstraram que as OR foram mais significativas na associação entre CC e hipertensão, diabetes e dislipidemias, principalmente em mulheres com menos de 40 anos de idade ${ }^{23}$. Foi observado também que a CC é um preditor independente de elevados escores de risco metabólico e resistência à insulina em mulheres chinesas pós-menopausa ${ }^{24}$.

A presença de associação entre indicadores antropométricos de obesidade e RCE, mais forte em mulheres mais jovens, pode ser explicado pela influência de outros fatores relacionados ao processo do envelhecimento, já que entre as mulheres mais idosas, em função da menopausa com conseqüentes modificações hormonais, outras variáveis que se tornam mais prevalentes nestas idades, como as dislipidemias, podem influenciar no aumento do risco coronariano.

De modo geral, observa-se que os indicadores de obesidade central ou abdominal estão mais associados ao risco coronariano aumentado do que os indicadores de obesidade total, independentemente da idade e do sexo. Isto provavelmente se deve ao fato de que o acúmulo excessivo de gordura abdominal está associado a diversas alterações metabólicas, tais como intolerância à glicose, hiperinsulinemia, diabetes, hipertensão e alterações nas concentrações de lipídios e lipoproteínas plasmáticas, geralmente presentes nas doenças coronarianas ${ }^{25}$.

O presente estudo, de corte transversal, tem as limitações que lhe são inerentes com a exposição e desfecho mensurados em determinado instante do tempo, dificultando a afirmação de relação causa-efeito em função da ambigüidade temporal.

Outra limitação foi o uso da classifica- ção do RCE feita através do algoritmo proposto com base na coorte de Framinghan ${ }^{3}$. Utilizamos este algoritmo aplicando seus valores nos indivíduos participantes da nossa amostra e definimos de forma arbitrária que o RCE seria identificado através da pontuação referente ao percentil 80 na distribuição de percentis da própria população de estudo. Existem controvérsias quanto à pontuação ideal para identificação do RCE em curto prazo. Alguns autores sugerem ${ }^{26}$ que o RCE poderia ser identificado através da pontuação equivalente a $20 \%$ do risco absoluto para coronariopatia nos próximos 10 anos, o que equivaleria a 9 pontos para homens e 15 pontos para mulheres no algoritmo proposto com base na coorte de Framinghan ${ }^{3}$. No nosso estudo, o RCE foi identificado através do ponto de corte de 8 pontos para homens e 10 pontos para mulheres.

Os resultados do presente estudo demonstram que, entre pessoas do sexo masculino, os indicadores de obesidade central (RCCQ e índice C) apresentam maiores associações com RCE. A CC apresenta associação um pouco menor, enquanto o IMC apresenta as menores e menos significativas associações com RCE. Entre pessoas do sexo feminino com idade entre 3049 anos, todos os indicadores antropométricos de obesidade apresentam associação com RCE, enquanto entre mulheres com idade variando entre 50-74 anos as associações são de menor magnitude, e apenas o índice $\mathrm{C}$ apresenta associação estatisticamente significativa com RCE. Considerando a forte modificação de efeito encontrada em pessoas do sexo feminino na variável idade, sugerem-se novos estudos para analisar a utilização dos indicadores antropométricos de obesidade como instrumento de triagem para RCE em mulheres de diferentes grupos etários. 


\section{Referências}

1. Pollock ML, Wilmore JH, Fox, SM. Exercícios na saúde e na doença: Avaliação e prescrição para prevenção e reabilitação. Editora Medsi: Rio de Janeiro; 1993.

2. James PT, Leach R, Kalamara E, Shayeghi, M. The worldwide obesity epidemic. Obesity Res 2001; 9(4): 228-33.

3. Wilson PWF, D'agostino RB, Levy D, Belanger AM, Silbershatz H, Kannel WB. Prediction of coronary heart disease using risk factores categories. Circulation 1998; 97: 1837-47.

4. Lessa I. O Adulto brasileiro e as doenças da modernidade: epidemiologia das doenças crônicas não-transmissíveis. Editora Hucitec-Abrasco: São Paulo; 1998.

5. Bose K, Mascie-Taylor CGN. Conicity index and waist-hip ratio and their relationship with total cholesterol and blood pressure in middle-age European and migrant Pakistani men. Ann Hum Biol 1998; 25(1): 11-6.

6. Venkatramana P, Reddy PC. Association of overall and abdominal obesity with coronary heart disease risk factors: comparison between urban and rural Indian men. Asia Pac J Clin Nutr 2002; 11(1): 66-71.

7. Ghosh A, Fitzgerald MH, Bose K, Chaudhuri AB. Association of food patterns, central obesity measure and metabolic risk factors for coronary heart disease (CHD) in middle age Bengalee Hindu men, Calcutta, India. Asia Pac JClin Nutr 2003; 12(2): 166-71.

8. Pitanga FJG, Lessa I. Indicadores antropométricos de obesidade como instrumento de triagem para risco coronariano elevado em adultos na cidade de SalvadorBahia. Arq Bras Cardiol 2005; 85(1): 26-31.

9. Bray GA. Pathophysiology of obesity. Am J Clin Nutr 1992; 55(2): 488-94.

10. Valdez R. A simple model-based index of abdominal adiposity. J Clin Epidemiol 1991; 44(9): 955-6.

11. Pouliot MC, Desprès JP, Lemieux S, Moorjani S, Bouchard C, Tremblay A, Nadeau A, Lupien PJ. Waist circumference and abdominal sagittal diameter: Best simple anthropometric indexes of abdominal visceral adipose tissue accumulation and related cardiovascular risk in men and women. Am J Cardiol 1994; 73: 460-8.

12. Vanltallie TB. Waist circunference: a useful index in clinical care and health promotion. Nut Rev 1998; 56(10): 300-2.

13. Brasil, Ministério da Saúde/Centro Nacional de Epidemiologia (CENEPI) - Instituto de Saúde Coletiva (ISC). Projeto Monitoramento de Doenças Cardiovasculares e do Diabetes no Brasil (MONIT). Relatório. Ministério da Saúde - CENEPI; 2000.

14. Teixeira MG, Barreto, ML, Costa MCN, Strina, A, Martins Júnior D, Prado, M. Áreas sentinelas: uma estratégia de monitoramento em saúde pública. Cad Saúde Pública 2002; 18(5): 1189-95.
15. Pitanga FJG, Lessa I. Sensibilidade e Especificidade do Índice de Conicidade como Discriminador do Risco Coronariano de Adultos em Salvador, Brasil. Rev Bras Epidemiol 2004; 7(3): 259-69.

16. Hosmer JR, Lemeshow S. Appled Logistic Regression. John Wiley \& Sons: New York: John Wiley \& Sons; 1989.

17. Coniglio RI, Colombo O, Vasquez L, Salgueiro AM, Otero JC, Malaspina MM. Relacion entre el indice de conicidad y los factores de riesgo lipoproteicos para la ateroesclerosis coronaria. Med 1997; 57(1):21-8.

18. Pitanga FJG, Lessa I, Araújo MJ, Magalhães L. Associação entre Îndice de Conicidade e Níveis de Glicose Plasmática. In: Resumos do 5 Congresso Brasileiro de Epidemiologia; 2002; Curitiba: ABRASCO; 2002. p. 360.

19. Pitanga FJG, Lessa I. Associação entre Índice de Conicidade e Hipertensão Arterial Sistólica em Adultos na Cidade de Salvador-Ba. In: Resumos do XIV Congresso de Cardiologia do Estado da Bahia; 2002; Salvador: Sociedade Brasileira de Cardiologia; 2002. p. 25.

20. Yasmin, Mascie-Taylor CGN. Adiposity indices and their relationship with some risk factores of coronary heart disease in middle-age Cambridge men and women. Ann Hum Biol 2000; 27(3): 239-48.

21. Richelsen B, Pedersen SB. Associations between different anthropometric measurements of fatness and metabolic risk parameters in non-obese, healthy, middle-age men. Int JObes Relat Metab Disord 1995; 19(3): 169-74.

22. Valdez R, Seidell JC, Ahn YI, Weiss KM. A new index of abdominal adiposity as na indicator of risk for cardiovascular disease. A cross-population study. Int J Obes Rel Met Disorders 1993; 17(2): 77-82.

23. Foucan L, Hanley J, Deloumeaux J, Suissa S. Body mass index (BMI) and waist circumference (WC) as screening tools for cardiovascular risk factors in Guadeloupean women. J Clin Epidemiol 2002; 55(10): 990-6.

24. Hwu CM, Fuh JL, Hsiao CF, Wang SJ, Lu SR, Wei MC, Kao WY, Hsiao LC, Ho LT. Waist circumference predicts metabolic cardiovascular risk in postmenopausal Chinese women. Menopause, 2003; 10(1): 73-80.

25. Després JP, Pouliot MC, Moorjani S, Nadeal A, Tremblay A, Lupien PJ, Thériault G, Bouchard C. Loss of abdominal fat and metabolic response to exercise training in obese women. Am J Physiol 1991; 261(24): E159-E67.

26. Grundy SM, Pasternak R, Greenland P, Smith S, Fuster V. Assessment of cardiovascular risk by use of multiple-riskfactor assessment equations. JAm Coll Cardiol 1999; 34: 1348-59.

Recebido em: 18/09/06 Versão final reapresentada em: 17/11/06 Aprovado em: 03/05/07 